\title{
Earthworm casting activities under bamboo plantations of West Tripura, India and their impact on soil physicochemical properties
}

\author{
Sourabh Chakraborty ${ }^{1}$, Niladri Paul ${ }^{2}$ and P. S. Chaudhuri ${ }^{1, *}$ \\ ${ }^{1}$ Earthworm Research Laboratory, Department of Zoology, Tripura University, Suryamaninagar, West Tripura 799 022, India \\ ${ }^{2}$ Department of Soil Science and Agricultural Chemistry, College of Agriculture, Tripura, Lembucherra, West Tripura 799210 , India
}

Earthworm casting activities under five species of bamboo plantations of West Tripura, India and their physicochemical relationship with the surrounding soil were studied for two successive years (2016 and 2017). Annual cast production in the bamboo plantations of West Tripura was $42,620 \mathrm{~kg} \mathrm{ha}^{-1}$ year $^{-1}$. Maximum casting activity occurs in summer followed by monsoon which is statistically higher than $(P<0.05)$ in winter. The amount of cast production and its monthly variation strongly correlated $(r=0.60, P=$ 0.008 ) and coincided with the amount and pattern of rainfall in West Tripura. The optimum temperature range for maximum casting was between $26^{\circ} \mathrm{C}$ and $27^{\circ} \mathrm{C}$. Amount of cast produced had strong positive correlation with soil moisture $(r=0.56, P=0.003)$ and negative correlation with soil temperature $(r=-0.55$, $\boldsymbol{P}=0.004)$. Casts in general were significantly richer $(P<0.05)$ in different physicochemical parameters (moisture, pH, available $\mathrm{N}, \mathrm{P}, \mathrm{K}$, etc.) than the surrounding soils.

Keywords: Bamboo plantations, cast morphometry, earthworm castings, edaphic factors, physico-chemical analysis.

BAMBOO plantations have gained considerable attention in recent years on account of having much higher carbon sequestration potential compared to other forest species $^{1-3}$. A large variety of wild weeds along with persistent availability of bamboo leaf litter in the plantation floor is a characteristic feature of bamboo plantations. Chakraborty and Chaudhuri ${ }^{4}$ reported 16 species of earthworms along with abundance of surface castings in the bamboo plantations of West Tripura, India. This indicates the substantial role of earthworm species in maintaining the nutrient dynamics of these low-input agroecosystems.

Earthworms enhance the incorporation of plant residues into soil aggregates and make the soil porous through their burrowing and casting activities that affect

*For correspondence. (e-mail: priya_1956@rediffmail.com) organic matter localization in the soil ${ }^{5,6}$. They convert biodegradable materials and the soils they feed upon into nutrient-rich vermicast. The casts egested by earthworms are considered to be more stable than the parent soil aggregates, and contain certain enzymes, hormones, microorganisms, inorganic and organic materials acquired during the passage of soil through earthworm gut ${ }^{7}$. As $99.9 \%$ of ingested material is egested as cast ${ }^{8}$, the latter is a good indicator of burrowing and soil turnover rate occurring in the soil ecosystems. Earthworm activity affects soil porosity and aggregate size distribution, stability, aeration and hydraulic conductivity ${ }^{9}$. Earthworms influence plant growth by changing the spatio-temporal availability of nutrients, mainly phosphorus, nitrogen and carbon in their casts and burrow wall ${ }^{9}$. Recently, Don et $a l{ }^{10}$ advocated the important role of anecic earthworms in increasing carbon sequestration and thereby accumulation in their vertical burrows that serve as fast ways for carbon transport in deep soil horizon. Chakraborty and Chaudhuri ${ }^{4}$ reported high density and biomass of phytogeophagous or anecic earthworms that produced surface casts under bamboo stands of Melocanna baccifera (locally called 'Muli' bamboo) and Bambusa bambos ('Katabarak' bamboo). Although casting activities of earthworms have been reported from various pedoecosystems of North East India such as jhum ${ }^{11}$, rubber ${ }^{12}$, pineapple $^{13}$ and tea plantations ${ }^{14}$, detailed reports on the casting activities of earthworms are lacking from bamboo plantations, which in fact act as a potential source of carbon sink in NE India ${ }^{15}$. Thus, the main objective of this study is to examine the casting activities of ten species of earthworms, viz. Drawida assamensis, Drawida papillifer papillifer, Eutyphoeus assamensis, Eutyphoeus comillahnus, Eutyphoeus scutarius, Eutyphoeus gammiei, Kanchuria sp. 1, Metaphire houlleti, Metaphire posthuma and Pontoscolex corethrurus in the soils of tropical bamboo ecosystems (Bambusa cacharensis, Bambusa polymorpha, Melocanna baccifera, Bambusa balcooa and Bambusa bambos) of West Tripura, and determine their correlation with various climatic and edaphic physicochemical parameters. Such a study would indicate the role of earthworms in nutrient cycling under low-input agro-ecosystems. 


\section{Materials and methods}

For studies on quantification of earthworm castings, cast morphometry and their relationship with various physicochemical factors, two sites for each species of bamboo plantation from various parts of West Tripura (total ten sites, viz. Madhuban, Ballavpur, Mahesh Khola, Syma Prashad, Brojendra Colony, Dyracherra, Madhya Dukli, Gabtali, Bholagiri and Durgapur) were selected. These study sites were $20-50 \mathrm{~km}$ apart from each other. The study areas experience a subtropical humid climate with a mean annual temperature of $25^{\circ} \mathrm{C}$ and annual rainfall above $2000 \mathrm{~mm}$, and four seasons, viz. summer (AprilJune), monsoon (July-October), winter (NovemberFebruary) and spring (March).

The studied bamboo species are mostly native to NE India (B. cacharensis, B. balcooa and B. bambos) or neighbouring Bangladesh (M. baccifera and B. polymorpha). B. polymorpha and B. balcooa mostly grow well in plain residential areas or river banks where they are used as embankments to reduce soil erosion, whereas $B$. $c a$ charensis, B. bambos and M. baccifera grow in undulating hills and hillocks of West Tripura. Species growing near residential areas and river banks are subjected to high anthropogenic interference and natural flooding during high rainfall periods, whereas species which grow on the slopes of hilly areas are subjected to least anthropogenic influence. Bamboo plantation floor usually harbours a wide variety of weeds and shrubs, viz. Mallotus philippensis (Euphorbiaceae), Eupatorium odoratum (Asteraceae), Grewia nervosa (Malvaceae), Olax acuminata (Olacaceae), Streblus asper (Moreceae), Calamus rotang (Arecaceae), Ziziphus rugosa (Rhamnaceae) and Chassalia curviflora (Rubiaceae).

\section{Sampling of earthworm casts}

Sampling of earthworm casts was done with two major objectives: (i) to study monthly variations in total cast productions in the bamboo plantations, and (ii) for species-specific morphometric studies on casts and their correlation with various climatic, physico-chemical and edaphic factors. Sampling was done on a monthly basis for each plantation starting from January 2016 to December 2017 , i.e. for a period of two years. Only those earthworm species whose surface casts were easily identifiable were considered for the present study.

For objective (i), fresh earthworm casts irrespective of species were collected from ten random quadrates of $1 \mathrm{~m}^{2}$ area from each of the two sites, of the five different bamboo plantations (100 random samples for each month). Annual cast production was obtained from total monthly collection of average mass of casts (dry weight $\mathrm{g} \mathrm{m}^{-2}$ month $^{-1}$ ) and converting the data in terms of $\mathrm{kg} \mathrm{ha}^{-1} \mathrm{yr}^{-1}$.
For objective (ii), only fresh earthworm casts were collected randomly with immediate extraction of earthworms exactly from the same point by digging and hand-sorting method in order to get casts voided by the same individual. Photographs of the casts and their morphometric measurements were taken. The length, diameter and fresh weight of the worm which voided the cast was recorded. The fresh casts and adjacent soils from which they were collected were put in separate polythene bags and taken to the laboratory for further analysis. Soil temperature at each sampling point was recorded using a soil thermometer.

\section{Physico-chemical analysis of casts and surrounding soils}

The physical parameters such as moisture and water holding capacity of the casts and their adjacent soils were determined by gravimetric wet weight method and soil core method $^{16}$ respectively. For determination of chemical parameters, the respective casts and soil samples were air-dried in laboratory, crushed and sieved to $2 \mathrm{~mm}$ particle size. $\mathrm{pH}$ and electrical conductivity (EC) of the respective samples were determined using electronic $\mathrm{pH}$ and electrical conductivity meter respectively. Total organic carbon was determined by Walkley and Black method $^{17}$. Total nitrogen was determined by FOSS Kjeltec method (modified Kjeldahl method) ${ }^{18}$. Available nitrogen was determined by alkaline potassium permanganate method ${ }^{19}$. Available phosphorus was extracted using Brey and Kurtz method ${ }^{20}$, and spectrophotometric estimation by drawing a standard curve. Available potassium and sodium were estimated by flame photometer method using neutral normal ammonium acetate as the extractant $^{18}$.

\section{Results and discussion}

\section{Cast morphometry, cast production and its relationship with various physico-chemical parameters}

Among the ten studied earthworm species, six (D. assamensis, D. papillifer papillifer, E. comillahnus, Kanchuria sp. $1, M$. houlleti and $P$. corethrurus) were common to the soils of all five bamboo plantation species and others had restricted distribution (E. scutarius in B. cacharensis and $B$. balcooa plantations, E. assamensis in $M$. baccifera plantations, E. gammiei in B. polymorpha plantations, and $M$. posthuma in B. balcooa plantations).

Table 1 provides the characteristics of the studied earthworm species, their casts and adjacent soils found in the bamboo plantations of West Tripura. The casts produced were of various shapes and sizes. Different species of Eutyphoeus produced tower-like casts with thin or thick convolutions. M. houlleti, D. assamensis and 


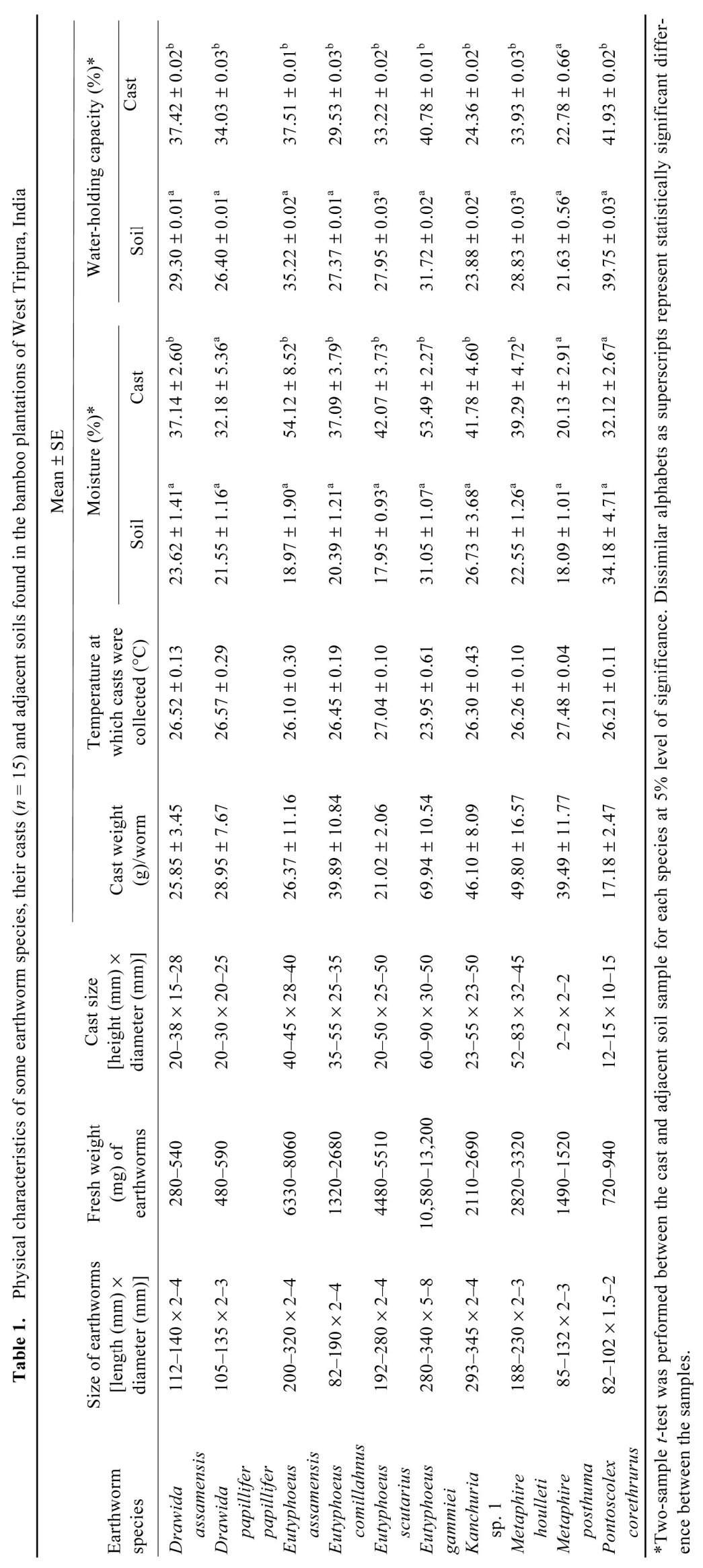




\section{RESEARCH ARTICLES}

Table 2. Relationship between different cast morphometric parameters, cast production and some physico-chemical parameters $(n=25)$

\begin{tabular}{lrrl}
\hline Parameters & $r$-value & $P$-value & Equation \\
\hline Dry cast weight $(\mathrm{g})$ versus organic carbon $(\mathrm{g})$ & -0.27 & 0.245 & Cast produced $=16.8938-6.14295 \times$ Organic carbon $(\mathrm{g})$ \\
Dry cast weight $(\mathrm{g})$ versus fresh worm weight $(\mathrm{g})$ & $\mathbf{0 . 5 7}$ & $\mathbf{0 . 0 0 3}$ & Cast produced $=27.7972+3.50904 \times$ Worm weight $(\mathrm{g})$ \\
Cast diameter $(\mathrm{cm})$ versus fresh worm weight $(\mathrm{g})$ & $\mathbf{0 . 4 8}$ & $\mathbf{0 . 0 1 2}$ & Cast diameter $=2.11945+0.500935 \times$ Worm weight $(\mathrm{g})$ \\
Cast production $\left(\mathrm{g} / \mathrm{m}^{2}\right)$ versus rainfall $(\mathrm{mm})$ & $\mathbf{0 . 6 0}$ & $\mathbf{0 . 0 0 8}$ & Cast produced $=153.649+0.735266 \times \mathrm{Rainfall}$ amount $(\mathrm{mm})$ \\
Dry weight of cast $(\mathrm{g})$ versus soil moisture $(\mathrm{g} \%)$ & $\mathbf{0 . 5 6}$ & $\mathbf{0 . 0 0 3}$ & Cast produced $=-15.7986+2.29542 \times$ Soil moisture $(\mathrm{g} \%)$ \\
Dry weight of cast $(\mathrm{g})$ versus soil temperature $\left({ }^{\circ} \mathrm{C}\right)$ & $-\mathbf{0 . 5 5}$ & $\mathbf{0 . 0 0 4}$ & Cast produced $=308.256-10.5417 \times$ Soil temperature $\left({ }^{\circ} \mathrm{C}\right)$ \\
\hline
\end{tabular}

$r$, Regression coefficient. Bold values represent statistically significant values at $5 \%$ level of significance.

D. papillifer papillifer had columnar casts with regular arrangement of spherical or sub-spherical aggregates. Casts of Kanchuria sp. 1 were large globoid mounds. $P$. corethrurus casts were composite, irregular, paste-like slurry, whereas $M$. posthuma casts comprised of mass of individual pallets. As defecating casts of $M$. posthuma were a collection of a large number of individual pellets, this is known as a decompacting species and others $(P$. corethrurus, $M$. houlleti, etc.) producing single, large biogenic structure are called compacting species. Although shape and size of casts vary with species, this cannot be taken as the sole criterion for the identification of earthworm species ${ }^{21}$. A probable relationship between casts of earthworms and their anal morphology was advocated by Singh ${ }^{22}$.

Table 2 shows the relationship between different cast morphometric parameters, cast production and some physico-chemical parameters. Earthworms contribute to soil aggregation mainly through the production of casts. In fact, earthworm casts contain more water-soluble aggregates than the surrounding soil $^{9}$. Cast production by earthworms in the bamboo plantations is mainly contributed by exotic species $P$. corethrurus and $M$. houlleti and native species $D$. assamensis and D. papillifer papillifer because of their dominance (relative abundance 12.5-40\%). Earthworms under bamboo plantations belong to the geophagous or endogeic community ${ }^{4}$. Due to low assimilation efficiency, these endogeic earthworms ingest and void large amounts of inorganic soil particles in the form of casts and thus play an important role with respect to soil turnover in the bamboo plantations. There was positive correlation between fresh earthworm weight with cast diameter $(r=0.48, P=0.012)$ and dry cast weight $(r=0.57, P=0.003)$. Usually size and weight of casts vary with the size of the earthworms. Thus large species such as E. gammiei produced large casts of 60 $90 \mathrm{~mm}$ height. M. houlleti produced 52-83 $\mathrm{mm}$ high casts. In the rubber plantations of Tripura, the largest cast was produced by large Octochaetid earthworm, E. gammiei $^{12}$. The large tower-like cast $(20-24 \mathrm{~cm}$ high and $4 \mathrm{~cm}$ diameter) of giant earthworm, Notosculex biramanicus from Burma weighted $1.6 \mathrm{~kg}$ after air-drying for four months ${ }^{21}$. The smallest cast produced by $M$. posthuma and $P$. corethrurus had the smallest body size and fresh weight among the studied species. There was a strong negative correlation between weight $(\mathrm{g})$ of casts produced and soil temperature $(r=-0.55, P=0.004)$ within the optimum range (Table 2 ). Fresh casts of most of the studied species were obtained at soil temperature range from $26.10^{\circ} \mathrm{C}$ to $27.48^{\circ} \mathrm{C}$ (Table 1 ). A strong positive correlation was observed between weight $(\mathrm{g})$ of cast produced and soil moisture $(r=0.56, P=0.003)$. Very weak negative correlation $(P>0.05)$ existed between amount of organic carbon in the soil and the amount of cast produced (Table 2).

Annual cast production in the bamboo plantations of West Tripura was $42,620 \mathrm{~kg} \mathrm{ha}^{-1} \mathrm{yr}^{-1}$. This was higher than that in tea plantations ${ }^{14} 37,380 \mathrm{~kg} \mathrm{ha}^{-1} \mathrm{yr}^{-1}$, mixed forest $\left(21,300 \mathrm{~kg} \mathrm{ha}^{-1} \mathrm{yr}^{-1}\right)$ and rubber plantations $\left(24,550 \mathrm{~kg} \mathrm{ha}^{-1} \mathrm{yr}^{-1}\right)$ of Tripura ${ }^{12}$. In the tropics, even larger amount of casts have been reported, ranging from $50,000 \mathrm{~kg} \mathrm{ha}^{-1}$ in Ghana ${ }^{23}, 507,000 \mathrm{~kg} \mathrm{ha}^{-1}$ in the Ivory Coast $^{24}$ to $2,100,000 \mathrm{~kg} \mathrm{ha}^{-1}$ in the Cameroons ${ }^{25}$. Darwin $^{26}$ estimated that annual production of worm casts in English pastures was $18,700-40,300 \mathrm{~kg} \mathrm{ha}^{-1}$. Thus the amount of soil turned over through casting activity differs greatly with habitat and geographical region ${ }^{9}$. The actual amount of soil turned over by the earthworms is perhaps even greater, as many species void their casts underground.

Figure 1 shows monthly variation in overall cast production in bamboo plantations of West Tripura along with rainfall pattern for the years 2016 and 2017. A strong positive correlation exists between cast production $\left(\mathrm{g} \mathrm{m}^{-2}\right)$, and both amount and pattern of rainfall $(r=0.60$, $P=0.008$ ) (Table 2). Cast production was almost nil from January to April, after which there was a rise from May reaching a peak in August which coincides with high rainfall (Figure 1). Hmar and Ramanujam ${ }^{27}$ recorded cast production in Mizoram from June to September with a peak in July. Bhadauria and Ramakrishnan ${ }^{28}$ found maximum cast production in the pine forests of Meghalaya during rainy season. As Tripura belongs to the highrainfall zone (rainfall $>2000 \mathrm{~mm} / \mathrm{yr}$ ), soil moisture is retained for a considerable period of time leading to sustained casting activities till the end of the year.

The number of casts produced varies seasonally and is a good index of earthworm activity ${ }^{29}$. Table 3 shows the monthly variations in cast production for 2016 and 2017 during the activity periods of the earthworms in the 
RESEARCH ARTICLES

Table 3. Variation in earthworm cast production $\left(\mathrm{g} \mathrm{m}^{-2}\right)$ under different bamboo plantations during 2016-2017 in West Tripura

\begin{tabular}{|c|c|c|c|c|c|c|c|c|c|}
\hline \multirow[b]{2}{*}{ Bamboo species } & \multicolumn{8}{|c|}{ Monthly average cast production $(n=20)$} & \multirow{2}{*}{$\begin{array}{c}\text { Average cas } \\
\text { production }\end{array}$} \\
\hline & May & June & July & August & September & October & November & December & \\
\hline B. cacharensis & $431.25^{\mathrm{acd}}$ & $395.85^{\text {acde }}$ & $511.91^{\mathrm{c}}$ & $269.90^{\text {ba }}$ & $251.16^{\mathrm{ba}}$ & $259.38^{\text {ba }}$ & $216.09^{\mathrm{eb}}$ & $102.17^{\mathrm{b}}$ & $304.71^{\mathrm{ab}}$ \\
\hline B. polymorpha & $545.45^{\mathrm{a}}$ & $929.58^{\mathrm{b}}$ & $538.31^{\mathrm{a}}$ & $474.40^{\mathrm{ac}}$ & $156.36^{\mathrm{ce}}$ & $79.17^{\mathrm{e}}$ & $108.72^{\text {ce }}$ & $127.33^{\mathrm{ce}}$ & $369.91^{\mathrm{ab}}$ \\
\hline M. baccifera & $289.51^{\mathrm{a}}$ & $212.12^{\mathrm{ac}}$ & $128.77^{\mathrm{bc}}$ & $102.78^{\mathrm{bc}}$ & $69.71^{\mathrm{bc}}$ & $114.72^{\mathrm{bc}}$ & $100.76^{\mathrm{bc}}$ & $309.18^{\mathrm{a}}$ & $165.94^{\mathrm{a}}$ \\
\hline B. balcooa & 364.12 & ND & ND & 231.57 & ND & ND & 69.38 & 33.59 & $174.66^{\mathrm{a}}$ \\
\hline B. bambos & $554.34^{\text {dac }}$ & $555.25^{\mathrm{dac}}$ & $809.89^{c}$ & $385.53^{\operatorname{deg}}$ & $673.99^{\mathrm{ac}}$ & $270.24^{\text {bef }}$ & $534.18^{\text {dace }}$ & $555.89^{\mathrm{acg}}$ & $542.41^{\mathrm{b}}$ \\
\hline
\end{tabular}

Dissimilar alphabets as superscripts signify statistically significant differences as shown by Tukey's post-hoc test. Results are at $5 \%$ level of significance. ND, not detected.

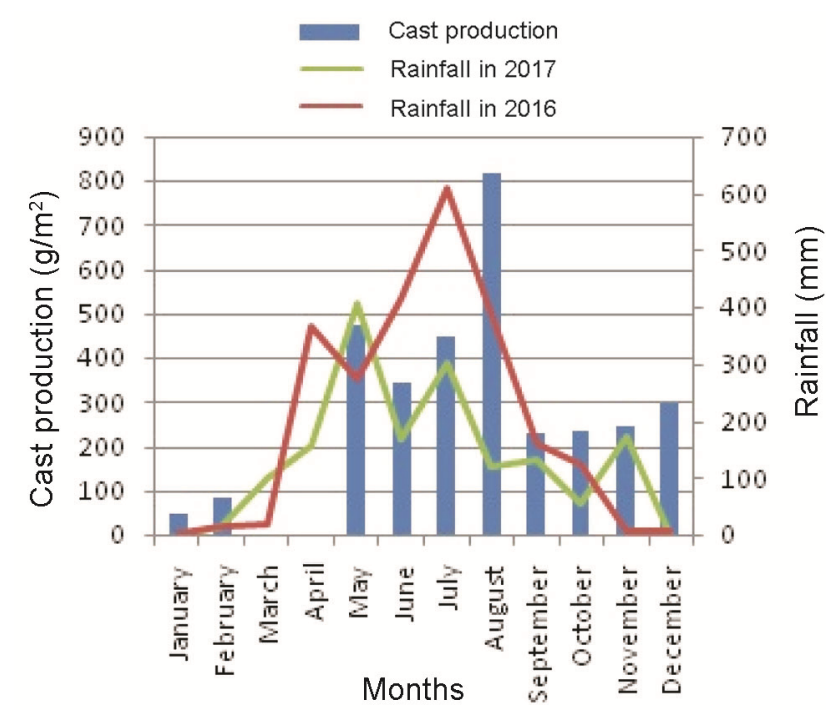

Figure 1. Overall month-wise average cast production in bamboo plantations of West Tripura, India along with rainfall pattern for 2016 and 2017.

plantations. Month-wise, there were some variations in casting activities from one plantation to another. Peak casting activities were observed in July for B. cacharensis and B. bambos plantations, whereas under B. polymorpha plantations, the highest casting activity was observed in June (Table 3). Interestingly, M. baccifera had the highest casting activities during May and December. The casting activities under B. balcooa were not determined every month because of severe anthropogenic activities and flooding of the plantations by the adjacent river water due to which discrete casts were not detected in certain months of the year.

Among the different seasons, cast production under different bamboo plantations varied significantly. Cast production in summer in all the plantations (B. cacharensis 413.55, B. polymorpha 737.51 , M. baccifera 250.81 and $B$. bambos $554.79 \mathrm{~g} \mathrm{~m}^{-2}$ ) was significantly higher $(P<0.05)$ than that during winter $(159.13,118.02$, 204.97 and $545.03 \mathrm{~g} \mathrm{~m}^{-2}$ respectively), but were at par $(P>0.05)$ during the monsoon season $(323.08,312.06$, 103.99 and $534.91 \mathrm{~g} \mathrm{~m}^{-2}$ respectively). The highest cast production was found under $B$. bambos plantations $\left(542.41 \mathrm{~g} \mathrm{~m}^{-2}\right.$ month $\left.^{-1}\right)\left(65,089.20 \mathrm{~kg} \mathrm{ha}^{-1}\right.$ year $\left.^{-1}\right)$ which was statistically much higher $(P<0.05)$ than in $M$. baccifera $\left(165.94 \mathrm{~g} \mathrm{~m}^{-2}\right.$ month $\left.^{-1}\right)\left(19,912.80 \mathrm{~kg} \mathrm{ha}^{-1} \mathrm{yr}^{-1}\right)$ and B. balcooa $\left(174.66 \mathrm{~g} \mathrm{~m}^{-2}\right.$ month $\left.^{-1}\right)\left(20,959.20 \mathrm{~kg} \mathrm{ha}^{-1} \mathrm{yr}^{-1}\right)$ plantations, but was at par with $B$. cacharensis (304.71 $\mathrm{g} \mathrm{m}^{-2}$ month $\left.^{-1}\right) \quad\left(36,565.20 \mathrm{~kg} \mathrm{ha}^{-1} \mathrm{yr}^{-1}\right)$ and $B$. polymorpha $\left(369.91 \mathrm{~g} \mathrm{~m}^{-2}\right.$ month $\left.^{-1}\right)\left(44,389.20 \mathrm{~kg} \mathrm{ha}^{-1} \mathrm{yr}^{-1}\right)$ plantations (Table 3). Differences in casting activities among different bamboo plantations are probably related to physico-chemical properties (moisture, temperature, texture) of soils, vegetation and earthworm assemblage ${ }^{30,31}$.

Thus variation in the amount of rainfall, soil moisture and soil temperature seem to be the key factor which regulates cast production by earthworms in the bamboo plantations of West Tripura.

\section{Comparison between physico-chemical parameters of cast and adjacent soil}

Physical properties of casts and their nutrient enrichment compared to the surrounding soils differed among the earthworm species probably due to differences in their gut-associated and cast-associated processes. Comparison of physical and chemical parameters with cast and adjacent soil is shown in Tables 1 and 4 respectively. In general, casts had much improved physico-chemical characteristics than their surrounding soils.

\section{Moisture}

Casts of seven out of ten studied earthworm species had significantly higher moisture content than their adjacent soils (Table 1). Highest amount of moisture was retained in casts of E. assamensis (54.12\%) followed by those of E. gammiei (53.49\%), whereas the least amount of moisture was obtained in the casts of $M$. posthuma (20.13\%). Compared to the surrounding soils, casts of E. assamensis retained a maximum of $35.15 \%$ more moisture, whereas $M$. posthuma casts had only $2 \%$ more moisture than their surrounding soils. Interestingly, casts of $P$. corethrurus retained less moisture content than their surrounding soils, although the differences were not statistically significant $(P<0.05)$. Retention of less moisture by earthworm casts 
Table 4. Chemical parameters of cast and adjacent soil $(n=3)$ of some earthworm species found in the bamboo plantations of West Tripura

\begin{tabular}{|c|c|c|c|c|c|c|c|c|c|c|}
\hline $\begin{array}{l}\text { Earth- } \\
\text { worm } \\
\text { species }\end{array}$ & $\begin{array}{c}D . \\
\text { assamensis }\end{array}$ & $\begin{array}{c}D . p . \\
\text { papillifer }\end{array}$ & $\begin{array}{c}\text { E. } \\
\text { assamensis }\end{array}$ & $\begin{array}{c}\text { E. } \\
\text { comillahnus }\end{array}$ & $\begin{array}{c}\text { E. } \\
\text { scutarius }\end{array}$ & $\begin{array}{c}\text { E. } \\
\text { gammiei }\end{array}$ & $\begin{array}{c}\text { Kanchuria } \\
\text { sp. } 1\end{array}$ & $\begin{array}{c}M . \\
\text { houlleti }\end{array}$ & $\begin{array}{c}M . \\
\text { posthuma }\end{array}$ & $\begin{array}{c}P . \\
\text { corethruru. }\end{array}$ \\
\hline \multicolumn{11}{|c|}{$\begin{array}{l}\text { Chemical parameters (mean } \pm \mathrm{SE})^{*} \\
\mathrm{pH}\end{array}$} \\
\hline Soil & $\begin{array}{c}4.86 \pm \\
0.01^{\mathrm{a}}\end{array}$ & $\begin{array}{c}5.03 \pm \\
0.01^{\mathrm{a}}\end{array}$ & $\begin{array}{c}4.72 \pm \\
0.01^{\mathrm{a}}\end{array}$ & $\begin{array}{c}4.81 \pm \\
0.01^{\mathrm{a}}\end{array}$ & $\begin{array}{c}5.22 \pm \\
0.01^{\mathrm{a}}\end{array}$ & $\begin{array}{c}5.29 \pm \\
0.01^{\mathrm{a}}\end{array}$ & $\begin{array}{c}4.63 \pm \\
0.03^{\mathrm{a}}\end{array}$ & $\begin{array}{c}4.96 \pm \\
0.01^{\mathrm{a}}\end{array}$ & $\begin{array}{c}5.92 \pm \\
0.01^{\mathrm{a}}\end{array}$ & $\begin{array}{c}5.49 \pm \\
0.01^{\mathrm{a}}\end{array}$ \\
\hline Cast & $\begin{array}{c}5.29 \pm \\
0.05^{\mathrm{b}}\end{array}$ & $\begin{array}{c}5.22 \pm \\
0.01^{\mathrm{b}}\end{array}$ & $\begin{array}{c}5.94 \pm \\
0.02^{\mathrm{b}}\end{array}$ & $\begin{array}{c}5.31 \pm \\
0.02^{\mathrm{b}}\end{array}$ & $\begin{array}{c}5.63 \pm \\
0.01^{\mathrm{b}}\end{array}$ & $\begin{array}{c}5.80 \pm \\
0.01^{\mathrm{b}}\end{array}$ & $\begin{array}{c}5.32 \pm \\
0.01^{\mathrm{b}}\end{array}$ & $\begin{array}{c}5.38 \pm \\
0.02^{\mathrm{b}}\end{array}$ & $\begin{array}{c}5.75 \pm \\
0.01^{\mathrm{a}}\end{array}$ & $\begin{array}{c}5.56 \pm \\
0.01^{\mathrm{a}}\end{array}$ \\
\hline \multicolumn{11}{|c|}{ EC (milli Mho/cm) } \\
\hline Soil & $\begin{array}{l}0.06 \pm \\
0.001^{\mathrm{a}}\end{array}$ & $\begin{array}{l}0.05 \pm \\
0.001^{\mathrm{a}}\end{array}$ & $\begin{array}{l}0.12 \pm \\
0.002^{\mathrm{a}}\end{array}$ & $\begin{array}{c}0.031 \pm \\
0.001^{\mathrm{a}}\end{array}$ & $\begin{array}{l}0.04 \pm \\
0.001^{\mathrm{a}}\end{array}$ & $\begin{array}{l}0.09 \pm \\
0.001^{\mathrm{a}}\end{array}$ & $\begin{array}{l}0.05 \pm \\
0.002^{\mathrm{a}}\end{array}$ & $\begin{array}{l}0.02 \pm \\
0.002^{\mathrm{a}}\end{array}$ & $\begin{array}{l}0.07 \pm \\
0.001^{\mathrm{a}}\end{array}$ & $\begin{array}{l}0.70 \pm \\
0.001^{\mathrm{a}}\end{array}$ \\
\hline Cast & $\begin{array}{l}0.62 \pm \\
0.001^{\mathrm{b}}\end{array}$ & $\begin{array}{l}0.11 \pm \\
0.001^{\mathrm{b}}\end{array}$ & $\begin{array}{l}0.26 \pm \\
0.002^{b}\end{array}$ & $\begin{array}{l}0.08 \pm \\
0.002^{\mathrm{b}}\end{array}$ & $\begin{array}{l}0.18 \pm \\
0.001^{\mathrm{b}}\end{array}$ & $\begin{array}{l}0.01 \pm \\
0.001^{\mathrm{b}}\end{array}$ & $\begin{array}{l}0.18 \pm \\
0.002^{\mathrm{b}}\end{array}$ & $\begin{array}{l}0.09 \pm \\
0.002^{\mathrm{b}}\end{array}$ & $\begin{array}{l}0.13 \pm \\
0.001^{\mathrm{b}}\end{array}$ & $\begin{array}{l}0.80 \pm \\
0.002^{\mathrm{b}}\end{array}$ \\
\hline \multicolumn{11}{|c|}{ Organic carbon $\left(\mathrm{g} \mathrm{kg}^{-1}\right)$} \\
\hline Soil & $\begin{array}{c}11.30 \pm \\
0.01^{\mathrm{a}}\end{array}$ & $\begin{array}{c}14.20 \pm \\
0.02^{\mathrm{a}}\end{array}$ & $\begin{array}{c}7.70 \pm \\
0.01^{\mathrm{a}}\end{array}$ & $\begin{array}{c}15.30 \pm \\
0.03^{\mathrm{a}}\end{array}$ & $\begin{array}{c}12.40 \pm \\
0.01^{\mathrm{a}}\end{array}$ & $\begin{array}{c}07.10 \pm \\
0.01^{\mathrm{a}}\end{array}$ & $\begin{array}{c}16.10 \pm \\
0.01^{\mathrm{a}}\end{array}$ & $\begin{array}{c}10.70 \pm \\
0.01^{\mathrm{a}}\end{array}$ & $\begin{array}{c}10.30 \pm \\
0.01^{\mathrm{a}}\end{array}$ & $\begin{array}{c}7.40 \pm \\
0.04^{\mathrm{a}}\end{array}$ \\
\hline Cast & $\begin{array}{c}24.20 \pm \\
0.02^{\mathrm{b}}\end{array}$ & $\begin{array}{c}24.70 \pm \\
0.01^{\mathrm{b}}\end{array}$ & $\begin{array}{c}22.70 \pm \\
0.01^{\mathrm{b}}\end{array}$ & $\begin{array}{c}21.50 \pm \\
0.01^{\mathrm{b}}\end{array}$ & $\begin{array}{c}24.70 \pm \\
0.01^{\mathrm{b}}\end{array}$ & $\begin{array}{c}22.30 \pm \\
0.01^{\mathrm{b}}\end{array}$ & $\begin{array}{c}20.60 \pm \\
0.02^{\mathrm{b}}\end{array}$ & $\begin{array}{c}11.30 \pm \\
0.01^{\mathrm{a}}\end{array}$ & $\begin{array}{c}11.70 \pm \\
0.02^{\mathrm{a}}\end{array}$ & $\begin{array}{c}15.30 \pm \\
0.03^{\mathrm{b}}\end{array}$ \\
\hline \multicolumn{11}{|c|}{ Total nitrogen $\left(\mathrm{kg} \mathrm{ha}^{-1}\right)$} \\
\hline Soil & $\begin{array}{c}2507.80 \pm \\
1.00^{\mathrm{a}}\end{array}$ & $\begin{array}{c}2821.41 \pm \\
0.99^{\mathrm{a}}\end{array}$ & $\begin{array}{c}2195.71 \pm \\
0.51^{\mathrm{a}}\end{array}$ & $\begin{array}{c}2509.00 \pm \\
0.51^{\mathrm{a}}\end{array}$ & $\begin{array}{c}1882.12 \pm \\
0.52^{\mathrm{a}}\end{array}$ & $\begin{array}{c}2195.21 \pm \\
0.01^{\mathrm{a}}\end{array}$ & $\begin{array}{c}3138.00 \pm \\
2.00^{\mathrm{a}}\end{array}$ & $\begin{array}{c}3449.61 \pm \\
0.01^{\mathrm{a}}\end{array}$ & $\begin{array}{c}2192.71 \pm \\
2.49^{\mathrm{a}}\end{array}$ & $\begin{array}{c}1882.61 \pm \\
1.01^{\mathrm{a}}\end{array}$ \\
\hline Cast & $\begin{array}{c}4391.89 \pm \\
1.49^{\mathrm{b}}\end{array}$ & $\begin{array}{c}4391.40 \pm \\
1.00^{\mathrm{b}}\end{array}$ & $\begin{array}{c}4076.31 \pm \\
0.48^{\mathrm{b}}\end{array}$ & $\begin{array}{c}3763.70 \pm \\
0.50^{\mathrm{b}}\end{array}$ & $\begin{array}{c}4076.82 \pm \\
0.02^{\mathrm{b}}\end{array}$ & $\begin{array}{c}3450.11 \pm \\
0.51^{\mathrm{b}}\end{array}$ & $\begin{array}{c}4705.00 \pm \\
1.00^{\mathrm{b}}\end{array}$ & $\begin{array}{c}2822.39 \pm \\
0.01^{\mathrm{b}}\end{array}$ & $\begin{array}{c}2822.50 \pm \\
0.10^{\mathrm{b}}\end{array}$ & $\begin{array}{c}3136.01 \pm \\
0.01^{\mathrm{b}}\end{array}$ \\
\hline \multicolumn{11}{|c|}{$\mathrm{C} / \mathrm{N}$ ratio } \\
\hline Soil & $\begin{array}{c}10.27 \pm \\
0.09^{\mathrm{a}}\end{array}$ & $\begin{array}{c}11.38 \pm \\
0.62^{\mathrm{a}}\end{array}$ & $\begin{array}{c}8.55 \pm \\
0.11^{\mathrm{a}}\end{array}$ & $\begin{array}{c}13.90 \pm \\
0.27^{\mathrm{a}}\end{array}$ & $\begin{array}{c}15.56 \pm \\
0.19^{\mathrm{a}}\end{array}$ & $\begin{array}{c}7.88 \pm \\
0.11^{\mathrm{a}}\end{array}$ & $\begin{array}{c}12.45 \pm \\
0.88^{\mathrm{a}}\end{array}$ & $\begin{array}{c}7.03 \pm \\
0.02^{\mathrm{a}}\end{array}$ & $\begin{array}{c}11.49 \pm \\
0.16^{\mathrm{a}}\end{array}$ & $\begin{array}{c}9.25^{\mathrm{a}} \pm \\
0.50\end{array}$ \\
\hline Cast & $\begin{array}{c}12.76 \pm \\
0.13^{\mathrm{b}}\end{array}$ & $\begin{array}{c}13.02 \pm \\
0.08^{\mathrm{a}}\end{array}$ & $\begin{array}{c}12.95 \pm \\
0.31^{\mathrm{b}}\end{array}$ & $\begin{array}{c}13.04 \pm \\
0.46^{\mathrm{a}}\end{array}$ & $\begin{array}{c}13.74 \pm \\
0.08^{\mathrm{b}}\end{array}$ & $\begin{array}{c}14.90 \pm \\
0.10^{\mathrm{b}}\end{array}$ & $\begin{array}{c}9.37 \pm \\
0.33^{\mathrm{a}}\end{array}$ & $\begin{array}{c}9.05 \pm \\
0.44^{\mathrm{b}}\end{array}$ & $\begin{array}{c}9.79 \pm \\
0.21^{\mathrm{b}}\end{array}$ & $\begin{array}{c}11.85^{\mathrm{a}} \pm \\
1.14\end{array}$ \\
\hline \multicolumn{11}{|c|}{ Available nitrogen $\left(\mathrm{kg} \mathrm{ha}^{-1}\right)$} \\
\hline Soil & $\begin{array}{c}440.05 \pm \\
1.01^{\mathrm{a}}\end{array}$ & $\begin{array}{c}375.76 \pm \\
0.55^{\mathrm{a}}\end{array}$ & $\begin{array}{c}313.50 \pm \\
0.10^{\mathrm{a}}\end{array}$ & $\begin{array}{c}437.54 \pm \\
1.49^{\mathrm{a}}\end{array}$ & $\begin{array}{c}251.88 \pm \\
1.00^{\mathrm{a}}\end{array}$ & $\begin{array}{c}376.35 \pm \\
0.05^{\mathrm{a}}\end{array}$ & $\begin{array}{c}314.10 \pm \\
0.5^{\mathrm{a}}\end{array}$ & $\begin{array}{c}440.03 \pm \\
0.99^{\mathrm{a}}\end{array}$ & $\begin{array}{c}188.66 \pm \\
0.50^{\mathrm{a}}\end{array}$ & $\begin{array}{c}250.81 \pm \\
0.01^{\mathrm{a}}\end{array}$ \\
\hline Cast & $\begin{array}{c}627.65 \pm \\
0.45^{\mathrm{b}}\end{array}$ & $\begin{array}{c}751.63 \pm \\
1.01^{\mathrm{b}}\end{array}$ & $\begin{array}{c}439.06 \pm \\
0.02^{\mathrm{b}}\end{array}$ & $\begin{array}{c}501.25 \pm \\
0.50^{\mathrm{b}}\end{array}$ & $\begin{array}{c}751.64 \pm \\
0.99^{\mathrm{b}}\end{array}$ & $\begin{array}{c}438.55 \pm \\
0.49^{\mathrm{b}}\end{array}$ & $\begin{array}{c}564.49 \pm \\
0.01^{\mathrm{b}}\end{array}$ & $\begin{array}{c}553.81 \pm \\
1.88^{\mathrm{b}}\end{array}$ & $\begin{array}{c}314.50 \pm \\
0.9^{\mathrm{b}}\end{array}$ & $\begin{array}{c}313.00 \pm \\
0.60^{\mathrm{b}}\end{array}$ \\
\hline \multicolumn{11}{|c|}{ Available phosphorus $\left(\mathrm{kg} \mathrm{ha}^{-1}\right)$} \\
\hline Soil & $\begin{array}{c}3.39 \pm \\
0.01^{\mathrm{a}}\end{array}$ & $\begin{array}{c}2.37 \pm \\
0.01^{\mathrm{a}}\end{array}$ & $\begin{array}{l}1.51 \pm \\
0.01^{\mathrm{a}}\end{array}$ & $\begin{array}{c}1.67 \pm \\
0.01^{\mathrm{a}}\end{array}$ & $\begin{array}{c}3.04 \pm \\
0.01^{\mathrm{a}}\end{array}$ & $\begin{array}{c}3.39 \pm \\
0.01^{\mathrm{a}}\end{array}$ & $\begin{array}{c}4.06 \pm \\
0.01^{\mathrm{a}}\end{array}$ & $\begin{array}{c}2.72 \pm \\
0.01^{\mathrm{a}}\end{array}$ & $\begin{array}{c}2.01 \pm \\
0.01^{\mathrm{a}}\end{array}$ & $\begin{array}{c}3.05 \pm \\
0.01^{\mathrm{a}}\end{array}$ \\
\hline Cast & $\begin{array}{c}4.41 \pm \\
0.01^{\mathrm{b}}\end{array}$ & $\begin{array}{c}1.34 \pm \\
0.01^{\mathrm{b}}\end{array}$ & $\begin{array}{c}1.54 \pm \\
0.01^{\mathrm{a}}\end{array}$ & $\begin{array}{c}2.72 \pm \\
0.01^{\mathrm{b}}\end{array}$ & $\begin{array}{c}4.05 \pm \\
0.01^{\mathrm{b}}\end{array}$ & $\begin{array}{c}3.40 \pm \\
0.05^{\mathrm{a}}\end{array}$ & $\begin{array}{c}2.70 \pm \\
0.05^{\mathrm{b}}\end{array}$ & $\begin{array}{c}3.04 \pm \\
0.01^{\mathrm{b}}\end{array}$ & $\begin{array}{c}3.39 \pm \\
0.05^{\mathrm{b}}\end{array}$ & $\begin{array}{c}3.06 \pm \\
0.01^{\mathrm{b}}\end{array}$ \\
\hline \multicolumn{11}{|c|}{ Available potassium $\left(\mathrm{kg} \mathrm{ha}^{-1}\right)$} \\
\hline Soil & $\begin{array}{c}218.30 \pm \\
0.10^{\mathrm{a}}\end{array}$ & $\begin{array}{c}211.80 \pm \\
1.00^{\mathrm{a}}\end{array}$ & $\begin{array}{c}368.49 \pm \\
0.01^{\mathrm{a}}\end{array}$ & $\begin{array}{c}236.31 \pm \\
0.01^{\mathrm{a}}\end{array}$ & $\begin{array}{c}167.00 \pm \\
1.00^{\mathrm{a}}\end{array}$ & $\begin{array}{c}459.70 \pm \\
0.5^{\mathrm{a}}\end{array}$ & $\begin{array}{c}220.62 \pm \\
0.02^{\mathrm{a}}\end{array}$ & $\begin{array}{c}191.51 \pm \\
0.01^{\mathrm{a}}\end{array}$ & $\begin{array}{c}179.65 \pm \\
0.45^{\mathrm{a}}\end{array}$ & $\begin{array}{c}167.60 \pm \\
0.40^{\mathrm{a}}\end{array}$ \\
\hline Cast & $\begin{array}{c}332.25 \pm \\
1.51^{\mathrm{b}}\end{array}$ & $\begin{array}{c}406.55 \pm \\
0.005^{\mathrm{b}}\end{array}$ & $\begin{array}{c}1692.31 \pm \\
0.01^{\mathrm{b}}\end{array}$ & $\begin{array}{c}385.79 \pm \\
0.51^{\mathrm{b}}\end{array}$ & $\begin{array}{c}349.94 \pm \\
0.5^{\mathrm{b}}\end{array}$ & $\begin{array}{c}484.93 \pm \\
0.03^{\mathrm{b}}\end{array}$ & $\begin{array}{c}325.89 \pm \\
0.03^{\mathrm{b}}\end{array}$ & $\begin{array}{c}360.62 \pm \\
0.02^{\mathrm{b}}\end{array}$ & $\begin{array}{c}296.70 \pm \\
0.10^{\mathrm{b}}\end{array}$ & $\begin{array}{c}210.54 \pm \\
0.002^{\mathrm{b}}\end{array}$ \\
\hline \multicolumn{11}{|c|}{ Available sodium $\left(\mathrm{kg} \mathrm{ha}^{-1}\right)$} \\
\hline Soil & $\begin{array}{c}119.82 \pm \\
0.02^{\mathrm{a}}\end{array}$ & $\begin{array}{c}100.70 \pm \\
0.10^{\mathrm{a}}\end{array}$ & $\begin{array}{c}169.11 \pm \\
0.01^{\mathrm{a}}\end{array}$ & $\begin{array}{c}118.74 \pm \\
0.02^{\mathrm{a}}\end{array}$ & $\begin{array}{c}97.42 \pm \\
0.02^{\mathrm{a}}\end{array}$ & $\begin{array}{c}81.73 \pm \\
0.03^{\mathrm{a}}\end{array}$ & $\begin{array}{c}119.82 \pm \\
0.02^{\mathrm{a}}\end{array}$ & ND & $\begin{array}{c}107.51 \pm \\
0.01^{\mathrm{a}}\end{array}$ & $\begin{array}{c}92.94 \pm \\
0.02^{\mathrm{a}}\end{array}$ \\
\hline Cast & $\begin{array}{c}114.22 \pm \\
0.02^{\mathrm{b}}\end{array}$ & $\begin{array}{c}108.62 \pm \\
0.02^{\mathrm{b}}\end{array}$ & $\begin{array}{c}403.22 \pm \\
0.02^{\mathrm{b}}\end{array}$ & $\begin{array}{c}103.02 \pm \\
0.02^{\mathrm{b}}\end{array}$ & $\begin{array}{c}117.70 \pm \\
0.1^{\mathrm{b}}\end{array}$ & $\begin{array}{c}118.71 \pm \\
0.01^{\mathrm{b}}\end{array}$ & $\begin{array}{c}98.57 \pm \\
0.01^{\mathrm{b}}\end{array}$ & ND & $\begin{array}{c}103.06 \pm \\
0.02^{\mathrm{b}}\end{array}$ & $\begin{array}{c}125.42 \pm \\
0.02^{\mathrm{b}}\end{array}$ \\
\hline
\end{tabular}

*Two-sample $t$-test was performed between the cast and the adjacent soil sample for each species at 5\% level of significance. Dissimilar alphabets as superscripts represent statistically significant difference between the samples. EC, Electrical conductivity. ND, Not determined.

of small size (M. postuma and P. corethrurus) is perhaps due to large exposed surface area of the casts compared to their volume, which might have led to rapid drying of the casts and consequently less moisture retention.

\section{Water-holding capacity}

Casts of all the earthworm species, except M. posthuma, had higher water-holding capacity than the surrounding soils $(P<0.05)$. The highest water-holding capacity was found in the casts of E. gammiei (40.78\%), which was about $9 \%$ higher than the surrounding soil (Table 1). Water-holding capacity is perhaps not correlated with cast size, since E. assamensis, E. comillahnus and Kanchuria sp. 1 despite having large-sized casts have less water-holding capacity compared to the surrounding soil, whereas species having smaller cast size, like $D$. assamensis and D. papillifer papillifer, have comparatively 
higher water-holding capacity. However, worms like E. gammiei and E. scutarius having large cast size also had higher water-holding capacity. Significant increase in water-holding capacity of casts than the neighbouring soil is probably due to abundance of micropores in the casts, which clearly indicates that earthworms influence moisture-holding capacity of soils which is an important factor for plant production ${ }^{9}$.

\section{$p H$}

Earthworm casts are usually more neutral than the soil in which the worms live ${ }^{9}$. Significant increase $(P<0.05)$ in $\mathrm{pH}$ of the casts of earthworm species (except $M$. posthu$m a$ ) (Table 4) from the acidic soils of bamboo plantations may result from excretion of ammonia into the intestine ${ }^{32}$ and/or production of calcium carbonate in calciferous glands and its release into the intestine ${ }^{33}$. Hmar and Ramanujam $^{27}$ also recorded higher $\mathrm{pH}$ in the casts than their surrounding soils in Mizoram. Contrary to these observations, Singh et al. ${ }^{7}$ observed $3.7 \%$ decrease in $\mathrm{pH}$ in the casts compared to surrounding alkaline soils in Punjab, India. Thus, earthworm activity acts as a buffer to maintain soil $\mathrm{pH}^{34}$.

\section{Electrical conductivity}

EC is a good indicator of the suitability of casts for agricultural purposes. Casts of all the studied species had substantially higher EC than their surrounding soils (Table 4). This is because casts are usually enriched with different mineral salts and ions because of selective feeding by the earthworms, which increase their $\mathrm{EC}^{12}$. Higher $\mathrm{EC}$ in the casts indicates an increase in the level of soluble salts in them over the surrounding soils. Higher EC in the casts has also been reported by Joshi and Kelkar ${ }^{35}$, and Reddy et $a l .{ }^{36}$. In contrast, Singh et $a l .^{7}$ reported significant decrease in EC from the soil to vermicast.

\section{Organic carbon}

Cast of all the studied species, except those of $M$. houlleti and $M$. posthuma had significant increase $(P<0.05)$ in the organic carbon content (Table 4$)$. High organic carbon in the casts compared to the surrounding soils has also been reported by earlier workers both in temperate $^{37,38}$ and tropical ${ }^{39}$ regions. This may partly be due to addition of intestinal mucus and also because earthworms may select soil fractions enriched in organic compounds $^{33,40}$. In fact, organic matter content provides stability to the casts 9 . The stabilization of earthworm casts increases with ageing and drying of the casts. In addition to organic matter humate synthesized in the earthworm intestine, calcium from calciferous gland, polysaccharide gum secreted by some bacteria and fungi probably contribute to the stabilization of earthworm casts ${ }^{9}$. Highest changes in organic carbon compared to the surrounding soils were observed in the casts of E. gammiei (3.14 times), E. assamensis (2.94 times), D. assamensis (2.14 times) and $P$. corethrurus (2.06 times). Although in $M$. houlleti and $M$. posthuma, organic carbon of the casts marginally increased compared to non-ingested soil, the change was not significant. This is probably due to greater carbon assimilation efficiencies of these species compared to the others.

\section{Total and available nitrogen}

Casts of all the studied species had significantly higher $(P<0.05)$ amount of both total and available nitrogen compared to the adjacent soils, except in $M$. houlleti where soil contained more total nitrogen than the casts (Table 4). In general, casts of most species had more than 1.5 times total nitrogen compared to the adjacent soils. Casts of M. houlleti, E. scutarius and D. papillifer papillifer had 3.14 times, 2.98 times and two times more available $\mathrm{N}$ compared to their surrounding soils.

Available N\% increased in the casts compared to that in the soils. It was $29.88,50.00,28.59,12.71,66.48$, $14.18,44.35,20.54,40.01$ and 19.86 in D. assamensis, $D$. papillifer papillifer, E. assamensis, E. comillahnus, E. scutarius, E. gammiei, Kanchuria sp. 1, M. houlleti, M. posthuma and P. corethrurus respectively. E. scutarius, D. papillifer papillifer and Kanchuria sp. 1 showed greater increase in the available nitrogen $(66.48 \%, 50 \%$ and $44.35 \%$ respectively) compared to their surrounding soils. This indicates that gut-associated processes in these species convert some of the unavailable forms of nitrogen from the total nitrogen pool into available nitrate nitrogen in the casts. The range of available nitrogen in comparison of total nitrogen in the casts in different earthworm species (D. assamensis 14.29\%, D. papillifer papillifer $17.11 \%$, E. assamensis $10.77 \%$, E. comillahnus $13.31 \%$, E. scutarius $18.43 \%$, E. gammiei $12.71 \%$, Kanchuria sp. 1 $11.99 \%$, M. houlleti $19.62 \%$, M. posthuma $11.14 \%$ and P. corethrurus $9.98 \%$ ) varies from about $10 \%$ to $20 \%$. $M$. houlleti, E. scutarius and D. papillifer papillifer had a higher percentage of available nitrogen compared to the other species, which may be due to their efficient gutassociated processes leading to effective unlocking of unavailable nitrogen from the total nitrogen pool. This increase in nitrogen in the casts might have occurred due to mucus (mucoprotein), nitrogenous excretory substances, hormones and nitrogen-fixating enzymes (nitrogenase) associated with the worm gut-associated processes $^{41}$. Earlier $\mathrm{Mba}^{42}$ reported that activity of nitrogen-fixing enzymes increased tenfold in Eudrilus eugeniae casts during composting of ground Dallas grass (Paspalum dilatatum). 


\section{$C: N$ ratio}

Among the ten species, casts of four (D. assamensis, E. assamensis, E. gammiei, M. houlleti) had increased $\mathrm{C}: \mathrm{N}$ ratio compared to their adjacent soils, whereas those of $E$. scutarius and $M$. posthuma had lower $\mathrm{C}: \mathrm{N}$ ratio than their surrounding soils (Table 4). Four other species had no significant difference compared to the adjacent soils. Hulugalle and Ezumah ${ }^{43}$ observed that earthworm casts of cassava-based cropping system in a tropical Alfisol had higher $\mathrm{C}: \mathrm{N}$ ratio than their surrounding soils. However, Mulongoy and Bedoret ${ }^{44}$ reported that casts of Alfisols had significantly lower $\mathrm{C}: \mathrm{N}$ ratio than their adjacent soils. Thus, differences in $\mathrm{C}: \mathrm{N}$ ratio between casts and soils as observed in this study may have occurred due to differences in soil properties and vegetation in which each species resides.

\section{Available phosphorus}

Out of the ten species, casts of six (D. assamensis, E. comillahnus, E. scutarius, M. houlleti, M. posthuma and $P$. corethrurus $)$ had significantly higher $(P<0.05)$ phosphorus content than their surrounding soils, and in the rest the differences were not significant (Table 4). Significant increase $(P<0.05)$ in available phosphorus in the casts relative to their surrounding soils is in agreement with the findings of other researchers ${ }^{7,12,45}$. The increase in phosphate content has been attributed to the presence of alkaline phosphatases in vermicast, as suggested by Prakash and Karmegam ${ }^{46}$.

\section{Available potassium and sodium}

All the cast samples had significantly higher $(P<0.05)$ levels of potassium compared to the adjacent soil samples (Table 4). Casts of E. assamensis, E. scutarius and D. papillifer papillifer especially had very high amounts of potassium compared to their surrounding soils $(78.22 \%$, $52.27 \%$ and $47.90 \%$ increase respectively). These findings are in agreement with those of Chaudhuri et al. ${ }^{12}$, who reported that significant increase in the concentration of available potassium in the casts of $M$. houlleti, Kanchuria sp. 1 and E. gigas was probably due to their selective feeding on foliar or other food materials enriched with this cation.

There were significant differences in available sodium $(P<0.05)$ between the cast samples and adjacent soil samples which, however, had no consistent pattern (Table 4). Sodium content was higher than the surrounding soils in the casts of D. papillifer papillifer, E. assamensis, E. scutarius, E. gammiei and P. corethrurus. However, in the casts of $D$. assamensis, E. comillahnus, Kanchuria sp. 1 and $M$. posthuma, it was lower than the surrounding soils. The effect the earthworms on cations like $\mathrm{Na}^{+}, \mathrm{K}^{+}$,
$\mathrm{Ca}^{++}$and $\mathrm{Mg}^{++}$had no consistent trend and was of lower magnitude than those from field soils, suggesting that elevated levels of cations often found in earthworm casts from field soils are probably due to selective feeding on materials rich in these cations by the earthworm species ${ }^{9}$.

\section{Conclusion}

Following feeding of soils, the earthworms produce egesta which are called casts. There are different forms of casts which are often species specific. Casts of earthworm species, in general, are richer in organic $\mathrm{C}, \mathrm{N}, \mathrm{P}$ and $\mathrm{K}$ than the surrounding soils. The present study showed that the amount of cast production in bamboo plantations strongly correlates and coincides with the amount and pattern of rainfall. As earthworm casts are water stable aggregates and are richer in plant nutrients, it may be assumed that earthworms in bamboo plantations have important role in soil carbon conservation and nutrient turnover.

Conflict of interest: The authors declare no conflict of interest.

1. Wang, B., Wang, Y., Guo, H., Zhao, G. D. and Bai, X. L., Carbon storage and spatial distribution in Phyllostachys pubescens forest in Dagangshan Mountain of Jiangxi province. J. Beijing For. Univ., 2009, 31, 39-42.

2. Yen, T. M. and Lee, J. S., Comparing aboveground carbon sequestration between moso bamboo (Phyllostachys heterocycla) and China fir (Cunninghamia lanceolata) forests based on the allometric model. For. Ecol. Manage., 2011, 261, 995-1002.

3. Zhou, G. M. and Jiang, P. K., Density, storage and spatial distribution of carbon in Phyllostchys pubescens forest. Sci. Silvae. Sin., 2004, 40, 20-25.

4. Chakraborty, S. and Chaudhuri, P. S., Earthworm communities in the bamboo plantations of West Tripura (India). Proc. Zool. Soc., 2017, 70, 105-118.

5. Ketterings, Q. M., Blair, J. M. and Marinissen, J. C. Y., Effects of earthworm on soil aggregate stability and carbon and nitrogen storage in legume cover crop agro ecosystem. Soil Biol. Biochem., 1997, 29, 401-408.

6. Bossuyt, H., Six, J. and Hendrix, P. F., Rapid incorporation of carbon from fresh residues into newly formed stable micro aggregates within earthworm casts. Eur. J. Soil Sci., 2004, 55, 393-399.

7. Singh, S., Singh, J. and Vig, A. P., Effect of abiotic factors on the distribution of earthworms in different land use patterns. J. Basic Appl. Zool., 2016, 74, 41-50.

8. Lavelle, P., Les vers de terre de la Savanne de Lamto, in Analyse d'un Ecosysteme Tropical Humide: La Savanne de Lamto (Cote d'Ivoire). Bull. de Liaison des Chercheurs de Lamto, No. Spec., 1974, 5, 133-136.

9. Edwards, C. A. and Bohlen, P. J., Biology and Ecology of Earthworms, Chapman and Hall, London, UK, 1996.

10. Don, A., Steing, B., Schoning, I., Pritisch, K., Joschko, M., Gleixner, G. and Schulze, E. D., Organic carbon sequestration in earthworm burrows. Soil Biol. Biochem., 2008, 40, 1803-1812.

11. Bhadauria, T. and Ramakrishnan, P. S., Earthworm population dynamics and contribution to nutrient cycling during cropping and fallow phases of shifting agriculture (Jhum) in north east India. J. Appl. Ecol., 1989, 26, 505-520. 
12. Chaudhuri, P. S., Nath, S., Pal, T. K. and Dey, S. K., Earthworm casting activities under rubber (Hevea brasiliensis) plantations in Tripura (India). World J. Agric. Sci., 2009, 5, 515-521.

13. Dey, A. and Chaudhuri, P. S., Earthworm community structure of pineapple (Ananas comosus) plantations under monoculture and mixed culture in West Tripura, India. Trop. Ecol., 2014, 55, 1-17.

14. Jamatia, S. K. S. and Chaudhuri, P. S., Earthworm community structure under tea plantation (Camellia sinensis) of Tripura (India). Trop. Ecol., 2017, 58, 105-113.

15. Nath, A. J. and Das, A. K., Carbon pool and sequestration potential of village bamboos in the agroforestry system of northeast India. Trop. Ecol., 2012, 53, 287-293.

16. Upadhayay, R. M. and Sharma, N. L., Manual of Soil, Plant, Water and Fertilizer Analysis, Kalyani Publishers, New Delhi, 2001

17. Walkley, A. and Black, I. A., Determination of organic carbon in soil. Soil Sci., 1934, 37, 29-38.

18. Jackson, M. L., Soil Chemical Analysis, Prentice Hall of India Pvt Ltd, New Delhi, 1973.

19. Subbiah, B. V. and Asija, G. L., A rapid procedure for the determination of available nitrogen in soils. Curr. Sci., 1956, 25, 259 260.

20. Brey, R. H. and Kurtz, L. T., Determination of total, organic and available forms of phosphorus in soils. Soil Sci., 1945, 59, 39-45.

21. Kale, R. D., Earthworm - Cinderella of Organic Farming, Prism Book Pvt Ltd, Bangalore, 1998.

22. Singh, S. M., SEM studies on the anal morphology of two species of earthworms. In Seventh International Symposium on Earthworm Ecology, Cardiff, UK, 2002, p. 215.

23. Nye, P. L., Some soil-forming processes in the humid tropics. IV. The action of soil fauna. J. Soil Sci., 1955, 6, 78.

24. Lavelle, P., Consommation annuelle d'une population naturelle de vers de terre (Millsonia anomala omodes, Acanthodrilidae: Oligochaetes) dans la savanne de Lamto (cote d'Ivoire). In Progress in Soil Zoology (ed. Vanek, J.), Academia Publishing House, Prague, Czech Republic, 1975, pp. 299-304.

25. Kollmannsperger, F., Lumbricidae of humid and arid regions and their effect on soil fertility. In VI Congr. Int. Sci. Sol. Rapp. C., 1956, pp. 293-297.

26. Darwin, C. R., The Formation of Vegetable Mould through the Action of Worms, Murray, London, UK, 1881.

27. Hmar, L. and Ramanujam, S. N., Earthworm cast production and physico-chemical properties in agroforestry system of Mizoram (India). Trop. Ecol., 2014, 55, 75-84.

28. Bhadauria, T. and Ramakrishnan, P., Population dynamics of earthworms and their activity in forest ecosystems of North-east India. J. Trop. Ecol., 1991, 7, 305-318.

29. Roy, S. K., Studies on the activities of earthworms. Proc. Zool. Soc., 1957, 10, 81-98.

30. Tomlin, A. D., Shiptalo, M. J., Edwards, W. M. and Protz, R., Earthworms and their influence on soil structure and infiltration. In Earthworm Ecology and Biogeography in North America (ed. Hendrix, P. F.), Lewis Publisher, Boca Raton, Florida, USA, 1995, pp. $159-183$.

31. Birang, M., Hauser, S., Brussard, L. and Norgrove, L., Earthworm surface casting activity on slash and burn cropped land and in undisturbed Chromolaena odorata and young forest fallow in southern Cameroon. Pedobiologia, 2003, 47, 811-818.

32. Wallwork, J. A., Earthworm Biology, Edward Arnold, London, UK, 1983.
33. Lee, K. E., Earthworms: Their Ecology and Relationships with Soil and Land Use, Academic Press, Sydney, Australia, 1985.

34. Singh, J. and Kaur, A., Vermicompost as a strong buffer and natural adsorbent for reducing transition metals, BOD, COD from industrial effluent. Ecol. Eng., 2015, 74, 13-19.

35. Joshi, N. V. and Kelkar, B. V., The role of earthworms in soil fertility. Indian J. Agric. Sci., 1952, 22, 189-196.

36. Reddy, M. V., Reddy, V. R., Balashouri, P., Kumar, V. P. K., Cogle, A. L., Yule, D. F. and Babu, M., Responses of earthworm abundance and production of surface casts and their physicochemical properties to soil management in relation to those of an undisturbed area on a semi-arid tropical Alfisol. Soil Biol. Biochem., 1997, 29, 617-620.

37. Scullion, J., Neale, S. and Philipps, L., Comparisons of earthworm populations and cast properties in organic arable rotations. Soil Use Manage., 2002, 18, 293-300.

38. Zhang, H. and Schrader. S., Earthworm effects on selected physical and chemical properties of soil aggregates. Biol. Fertil. Soils, 1993, 15, 229-234.

39. Asawalam, D. O., Influence of cropping intensity on the production and properties of earthworm casts in a Leucaena alley cropping system. Biol. Fertil. Soils, 2006, 42, 506-512.

40. Blair, J. M., Parmelee, R. W. and Lavelle, P., Influences of earthworms on biogeochemistry. In Earthworm Ecology and Biogeography in North America (ed. Hendrix, P. F.), Lewis Publishers, Chelsea, UK. 1994, pp. 127-158.

41. Garg, V. K., Kaushik, P., Vermistabilization of textile mill sludge spiked with poultry droppings by epigeic earthworm Eisenia Fetida. Bioresour. Technol., 2005, 96, 1063-1071.

42. Mba, C., Vermicomposting and biological N-fixation. In Proceedings of the 9th International Symposium on Soil Biology and Conservation of the Biosphere (ed. Szegi, J.), Akad'emiai Kiado'o, Budapest, Hungary, 1987, pp. 547-552.

43. Hulugalle, N. R. and Ezumah, H. C., Effects of cassava based cropping systems on physico-chemical properties of soil and earthworm casts in a tropical Alfisol. Agric. Ecosyst. Environ., 1991, 35, 55-63

44. Mulongoy, K. and Bedoret, A., Properties of worm casts and surface soils under various plant covers in the humid tropics. Soil Biol. Biochem., 1989, 21, 197-203.

45. Bisht, R., Pandey, H., Bisht, S. P. S., Kandpal, B. and Kaushal, B. R., Feeding and casting activities of the earthworm (Octolasion tyrtaeum) and their effects on crop growth under laboratory conditions. Trop. Ecol., 2006, 47, 291-294.

46. Prakash, M. and Karmegam, N., Dynamics of nutrients and microflora during vermicomposting of mango leaf litter (Mangifera indica) using Perionyx ceylanensis. Int. J. Global Environ. Issues, 2010, 10, 339-353.

ACKNOWLEDGEMENT. We thank the Head, Department of Zoology, Tripura University and the Principal, College of Agriculture, Tripura for providing the necessary laboratory facilities.

Received 4 January 2019; revised accepted 1 July 2020

doi: $10.18520 / \mathrm{cs} / \mathrm{v} 119 / \mathrm{i} 7 / 1169-1177$ 